\title{
Efeito agudo de uma sessão de treinamento de futsal na modulação autonômica cardíaca de jovens jogadores
}

http://dx.doi.org/10.11606/1807-5509201700030527

\author{
Ana Carolina PALUDO* \\ Felipe Nunes RABELO** \\ Bernardo MILOSKI* \\ Enio Ricardo Vaz RONQUE ${ }^{* * *}$ \\ Helio SERASSUELO JUNIOR ${ }^{* * * *}$ \\ Antônio Carlos SIMÕES* \\ *Escola de Educação \\ Física e Esporte \\ Universidade de São \\ Paulo, São Paulo, SP, \\ Brasil. \\ ${ }^{* *}$ Clube Atlético \\ Paranaense, Curitiba \\ Paraná, Brasil. \\ ${ }^{* * *}$ Centro de \\ Educação Física e \\ Esporte, Universidade \\ Estadual de Londrina, \\ Londrina, PR, Brasil.
}

\section{Resumo}

0 objetivo do presente estudo foi avaliar o efeito de uma sessão de treinamento de futsal na modulação autonômica cardíaca (pré versus pós) de jovens atletas. Foram avaliados nove atletas de futsal do sexo masculino da categoria Sub 20 de um clube profissional do estado de São Paulo- Brasil (idade:18,9 $\pm 0,9$ anos, massa corporal:73,1 $\pm 6,0 \mathrm{~kg}$ e estatura: $179,0 \pm 0,1 \mathrm{~cm})$. A sessão de treinamento caracterizou-se como técnico/tática. A modulação autonômica cardíaca foi analisada pelos índices da variabilidade da frequência cardíaca (VFC) avaliada com registro contínuo da frequência cardíaca (Polar Team2 Pro) 15 minutos antes e após a sessão de treinamento, sendo os dados posteriormente exportados para o programa Kubios (2.0) para o cálculo dos índices: MRR, RMSSD, LFlog e HFlog. A carga de treino foi avaliada pelos métodos de PSE, TRIMP e PSE da sessão (sPSE). As mudanças pré e pós foram avaliadas pelo o teste $t$ de Student e a variação percentual $(P<0,05)$. Todos os índices apresentaram um decréscimo significativo após sessão de treinamento com variação percentual foi: 10,7; 26,9; 4,2 e 11,3\%, respectivamente, para os índices MRR, RMSSD, LFlog e HFlog. Variação percentual do índice LFlog apresentou correlação significativa $(P<0,05)$ com o TRIMP da sessão $(r=0,694)$. Conclui-se que uma única sessão de treinamento de futsal foi capaz de gerar mudanças significativas na modulação autonômica cardíaca de jovens jogadores de futsal, com uma variação de 4,2\% a 26,9\%. Essa variação esta positivamente correlacionada com a carga de treino. Essas informações podem auxiliar a comissão técnica no planejamento das sessões de treino, no monitorando das cargas de treinamento.

Palavras-Chave: Esporte; Variabilidade da Frequência Cardíaca; Carga de Treino; Monitoramento.

\section{Introdução}

Estímulos gerados pelo treinamento físico podem promover adaptações funcionais no sistema na modulação cardíaca autonômica relacionados ao aumento da aptidão física e ao desempenho esportivo demonstrados em diferentes esportes ${ }^{1,2}$, tanto de maneira aguda ${ }^{3,4}$, quanto cronicamente ${ }^{5,6}$. Desta forma, atletas que apresentam melhorias no componente cardíaco autonômico durante uma temporada tendem a exibir uma melhora em seu desempenho físico e específico da modalidade ${ }^{6}$.

A resposta autonômica ao treinamento pode ser monitorada através de medidas da variabilidade da frequência cardíaca (VFC). A VFC é uma ferramenta não invasiva, acessível, de baixo custo e fácil de ser utilizada no meio esportivo, tendo como objetivo monitorar a modulação autonômica cardíaca e os componentes simpático e vagal do sistema nervoso autônomo (SNA) ${ }^{7}$.

$\mathrm{O}$ registro da VFC no treinamento esportivo pode ser utilizado para avaliação de adaptações/ mal adaptações das sessóes de treino, além de ser utilizado no ajuste das cargas de treinamento (CT) visando o aumento do desempenho $0^{6,8,9,10}$. Além disso em uma sessão de treinamento, a VFC é capaz de 
prover informaçóes a respeito da magnitude das mudanças no nível de aptidão/fadiga dos atletas ${ }^{11,12}$, bem como auxiliar a equipe técnica na preparação das próximas sessôes de treinamento no que diz respeito a intensidade e/ou períodos de recuperação ${ }^{13}$.

Alguns estudos mensuraram as mudanças diárias nos valores dos índices de VFC com o objetivo de monitorar a resposta autonômica em função das CT e sua possível influência no desempenho esportivo na fase preparatória em corredores de elite ${ }^{12} \mathrm{e}$ na fase competitiva de jovens futebolistas ${ }^{3}$. Esses estudos aplicaram como metodologia a avaliação da VFC ao final de cada sessão de treinamento, comparando assim a variação diária ${ }^{3}$ e a mudança pré e pós uma temporada ${ }^{12}$, demonstrando que as mudanças autonômicas registradas diariamente estáo relacionadas com mudanças na máxima velocidade aeróbia e VO2 max $^{3,12}$, sendo capaz de predizer o desempenho esportivo $^{12}$. Entretanto, há uma escassez de estudos na literatura científica envolvendo a modalidade futsal, principalmente no que diz respeito ao monitoramento das respostas autonômicas ao treinamento.

O futsal é um esporte coletivo intermitente que permite ilimitadas substituiçóes durante o jogo, apresentando elevada demanda fisiológica similar ou superior a outros esportes coletivos ${ }^{14,15}$. Assim, tem sido proposto que a aptidáo aeróbia e a habilidade de realizar açóes repetidas de alta intensidade são componentes importantes para o desempenho competitivo dos jogadores ${ }^{6,16,17}$, uma

\section{Método}

\section{Amostra}

Fizeram parte do estudo nove jogadores de futsal do sexo masculino $(18,9 \pm 0,9$ anos, massa corporal $73,1 \pm 6,0 \mathrm{~kg}$ e estatura $179,0 \pm 0,1 \mathrm{~cm})$ da categoria Sub-20, de uma equipe de elite $(10,44 \pm 1,8$ anos de prática na modalidade, participante de campeonato regional e nacional) de São Paulo, Brasil. A equipe encontrava-se no final do período competitivo, no qual treinavam cinco vezes por semana. Todos os participantes assinaram voluntariamente um Termo de Consentimento Livre e Esclarecido (TCLE) assentindo sua participaçáo no estudo, sendo este aprovado no Comitê de Ética e Pesquisa local ( ${ }^{\circ}$ 544.5410). Todos os sujeitos não continham vez que a atividade máxima ou perto da máxima ocorre repetidamente durante o jogo, colaborando com as açóes decisivas na modalidade ${ }^{18}$. Pelo fato da modalidade apresentar estas características durante a maior parte do jogo, os atletas são expostos a uma elevada CT durante a temporada ${ }^{10,19,20}$.

Sendo assim, ressalta-se a importância do monitoramento das CT nesta modalidade, sobretudo em relação as mudanças ocorridas no sistema autonômico, que podem prover informações das adaptações funcionais geradas pelo estímulo do treinamento ${ }^{21}$. Alguns estudos sugerem a avaliação da CT por métodos baseados na frequência cardíaca ou percepção do esforço para o uso diário e aplicações práticas ${ }^{22}$, entretanto o uso das medidas de VFC apresentam-se como indicadores sensíveis a perturbaçôes homeostáticas como fadiga e estresse psicofisiológico ${ }^{1,23}$.

Respostas autonômicas geradas por uma única sessão de treinamento de futsal ainda são desconhecidas. Assim, não se sabe se as medidas de VFC são sensíveis ao estresse psicofísico imposto por uma sessão de treinamento de futsal. Isto posto, o objetivo do presente estudo foi examinar as respostas cardíacas autonômicas agudas decorrentes de uma sessão de treinamento utilizando as medidas de VFC bem como comparar a magnitude das mudanças cardíacas autonômicas com os dois métodos amplamente utilizados pelos técnicos para quantificar a CT. histórico de doença cardiopulmonar que pudessem influenciar o sistema nervoso autonômico.

\section{Delineamento do estudo}

O estudo foi conduzido durante uma única sessão de treinamento. A VFC foi registrada durante 15 minutos antes e depois da sessáo de treinamento pelo Polar Team ${ }^{2}$ Pro dentro do vestiário do próprio clube, estando os atletas na posição sentada e com respiração espontânea. No final da sessão de treinamento, a percepção subjetiva do esforço (PSE) da sessão foi registrada utilizando-se a escala de BorG $^{24}$. O delineamento é representado pela FIGURA 1. 


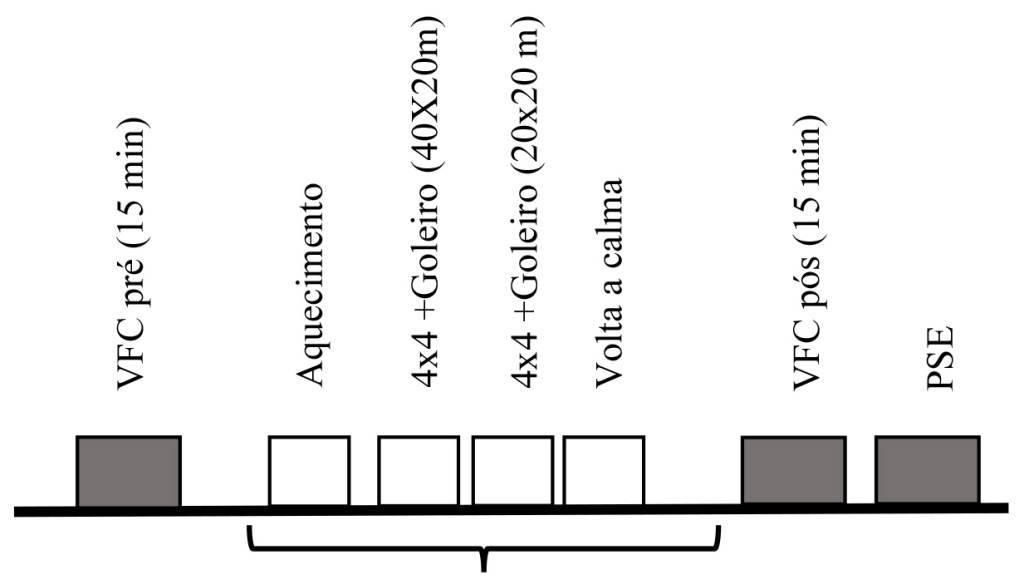

Sessão de Treinamento

FIGURA 1 - Esquema do delineamento experimental.

\section{Sessão de treinamento}

Antes da sessão de treinamento técnico/tático (16:00 - 18:00 h) os jogadores realizaram um aquecimento padronizado com duração de 15 minutos constituído de: alongamento dinâmico, exercícios de estabilização, mobilidade articular e exercícios de agilidade. Na primeira parte da sessão principal foi realizado um jogo 4 contra $4+$ goleiro (40x20m) com objetivo de desenvolver os princípios de transiçôes ofensivas e defensivas. Na segunda parte da sessão de treinamento realizou-se um jogo 4 contra 4 + goleiro em meia quadra $(20 \times 20 \mathrm{~m}) \mathrm{com}$ o objetivo de aperfeiçoar os princípios de ataque e estratégias defensivas baixas.

\section{Carga de treinamento}

O método de PSE da sessão foi utilizado para quantificar a carga de treinamento como proposto por Foster et al. ${ }^{25}$ PSE da sessão foi registrada cerca de 30 minutos depois do final da sessão de treinamento conforme estabelecido por estudos previos $^{24}$. Os atletas reportaram a PSE referente a intensidade da sessão de treinamento como um todo utilizando a escala de Borg CR-10. O escore de PSE (sPSE) registrado foi multiplicado pela duração da sessão, em minutos, para indicar a carga de treinamento ${ }^{25}$. O escore da PSE da sessão também foi retido para análise. Adicionalmente, calculouse o TRIMP da FC, a partir do método proposto por BANISTER ${ }^{26}$ utilizando a seguinte equação: Duração $\times \triangle F C \times 0.64^{\mathrm{e}} 1.92 x$ na qual o $\triangle \mathrm{FC}$ é igual a $\mathrm{FC}_{\text {exerćício }}-\mathrm{FC}_{\text {repouso }} / \mathrm{FC}_{\text {máxima }}-\mathrm{FC}$ respouso $_{\text {é } 1,92}$ diz respeito a constante da equação para homens. A frequência cardíaca máxima foi calculada pela equação de KARVONEN ${ }^{27}$ 220-idade.

\section{Avaliação autonômica}

As respostas cardíacas autonômicas foram avaliadas pelos índices de VFC pré e pós treinamento. A VFC foi registrada usando um cinto de frequência cardíaca Polar Team²Pro no tórax, com registro dos intervalos R-R. Os atletas encontravam-se sentados em uma cadeira durante 15 minutos antes e 15 minutos após a sessão de treinamento, no qual permaneceram quietos, com olhos abertos e respiração voluntária. Os índices de VFC foram calculados com base nos últimos 5 minutos de registros, sendo extraídos posteriormente como text file para o software Kubios (2.0) para análise dos parâmetros de VFC no domínio do tempo: média dos intervalos R-R (MRR), média raiz media quadrática da diferença entre os intervalos RR sucessivos (RMSSD); e análise no domínio da frequência: baixa e alta frequência em unidades de log (LFlog e HF log respectivamente), pelo falo da assimetria da distribuição dos valores ${ }^{28}$.

\section{Análise estatística}

Os dados descritivos são apresentados em média e desvio padrão uma vez que sua normalidade foi confirmada pelo teste de Shapiro Wilk. As diferenças entre pré e pós treinamento foram comparadas usando o teste "t" de Student e a magnitude das 
diferenças foram exibidas em variação percentual. A correlação de Pearson foi utilizada para verificar a associação entre as mudanças percentuais da VFC e a carga de treinamento. A análise qualitativa dos coeficientes de correlação foi realizada seguindo os parâmetros propostos por Hopkins ${ }^{29}$ : 0-0,09 trivial; 0,1-0,29 pequeno; 0,3-0,49 moderado; 0,5-0,69 grande; 0,7-0,89 muito grande e 0,90,99 quase perfeito. Para o tratamento dos dados utilizou-se o pacote estatístico SPSS (v.19) e o nível de significância foi estabelecido em $5 \%(P<0,05)$.

\section{Resultados}

A sessão de treinamento apresentou uma duração de 120 min., as características de carga de treinamento de cada atleta são apresentadas na TABELA 1.

A TABELA 2 apresenta os valores de VFC pré e pés sessão de treinamento para os atletas de futsal. Todos as medidas de VFC apresentaram um decréscimo significativo 15 min pós treino.

A magnitude da variação (pré-pós treino) das medidas de VFC são apresentados na FIGURA 2. A variação apresentou uma amplitude de $-4,2$ a $-26,9 \%$.

A correlaçáo entre os índices de VFC e os métodos de avaliação de CT (PSE; TRIMP, sPSE) apresentamse descritas na TABELA 3. As correlaçóes variaram de pequena a grande. A única correlação significativa (classificada como grande) foi a associação entre o índice LFlog e o TRIMP.

TABELA 1 - Característica do treinamento.

\begin{tabular}{lcccc}
\hline & PSE $(\mathbf{a u})$ & FC $\mathbf{m a x}(\mathbf{b} \mathbf{p m})$ & TRIMP $(\mathbf{a u})$ & sPSE (au) \\
\hline Atleta $\mathbf{1}$ & 6 & 184 & 42,12 & 720 \\
\hline Atleta $\mathbf{2}$ & 7 & 190 & 41,18 & 840 \\
\hline Atleta 3 & 7 & 188 & 56,49 & 840 \\
\hline Atleta 4 & 7 & 193 & 65,55 & 840 \\
\hline Atleta 5 & 7 & 183 & 71,61 & 840 \\
\hline Atleta 6 & 6 & 189 & 54,59 & 720 \\
\hline Atleta 7 & 6 & 187 & 58,09 & 720 \\
\hline Atleta 8 & 5 & 173 & 52,20 & 600 \\
\hline Atleta 9 & 8 & 182 & 53,63 & 960 \\
\hline Média & $\mathbf{6 , 5 6}$ & $\mathbf{1 8 5 , 4 4}$ & $\mathbf{5 7 , 1 2}$ & $\mathbf{7 8 6 , 6 7}$ \\
\hline ×DP & $\mathbf{0 , 8 8}$ & $\mathbf{5 , 8 5}$ & $\mathbf{6 , 5 6}$ & $\mathbf{1 0 5 , 8 3}$ \\
\hline
\end{tabular}

TABELA 2 - Respostas autonômicas pré e pós sessão de treinamento apresentadas em média (DP) em jogadores de futsal (n=9).

\begin{tabular}{lccc}
\hline & Pré & Pós & P \\
\hline MRR (ms) & $\begin{array}{c}786,7 \\
(157,7)\end{array}$ & $\begin{array}{c}741,2 \\
(137,3)\end{array}$ & 0,015 \\
\hline RMSSD (ms) & $\begin{array}{c}30,8 \\
(21,2)\end{array}$ & $\begin{array}{c}23,8 \\
(6,3)\end{array}$ & 0,015 \\
\hline $\operatorname{LF}(\log )$ & 8,0 & 7,0 & 0,025 \\
\hline HF $(\log )$ & $(1,0)$ & $(0,0)$ & 0,020 \\
\hline
\end{tabular}




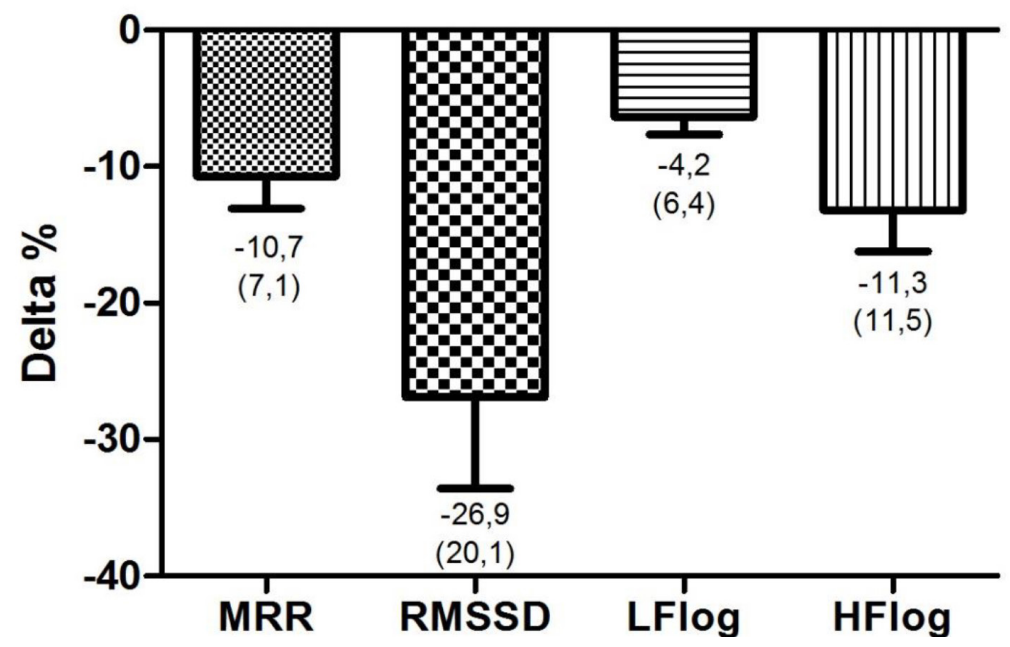

FIGURA 2 - Delta percentual (pré versus pós sessão de treinamento) das medidas de VFC.

TABELA 3 - Correlação (r) entre o percentual de mudança da VFC e métodos de CT.

\begin{tabular}{lccc}
\hline & PSE (au) & TRIMP (au) & sPSE \\
\hline MRR $(m s)$ & 0,579 & 0,460 & 0,579 \\
\hline RMSSD $(\mathrm{ms})$ & 0,286 & 0,550 & 0,286 \\
\hline LF $(\log )$ & $-0,263$ & $0,694^{*}$ & $-0,263$ \\
\hline HF $(\log )$ & 0,179 & 0,471 & 0,179 \\
\hline
\end{tabular}

\section{Discussão}

O objetivo do presente estudo foi examinar as respostas agudas decorrentes de uma sessão de treinamento, utilizando as medidas de VFC, bem como correlacioná-las com os métodos de avaliação de CT em jovens jogadores de futsal. O principal achado constituiu no decréscimo significativo nas medidas de VFC após a sessão de treinamento, mostrando-se sensíveis a demanda psicofisiológica imposta por uma sessão de treinamento de futsal. Além disso, este decréscimo apresentou correlação positiva e significativa do índice LFlog, na qual reflete a modulação vagal e simpática sobre o coraçãa ${ }^{30}$, com o método TRIMP de quantificação da CT.

Quando confrontados com a literatura, nossos resultados mostraram-se contraditórios com o estudo de RACZAK et al..$^{31}$ no qual avaliaram jovens (20 2 anos) durante uma sessão de exercício incremental em esteira rolante por 30 minutos a $65 \%$ FCmáx, não demonstrando diferença no pré e pós teste para os índices RMSSD e pNN50. Entretanto nossos resultados corroboram com os achados de SABOul et al. ${ }^{13}$, no qual encontraram diferença significativa para $o$ índice RMSSD após 4 sessôes de treinamento de corrida em diferentes intensidades em 11 corredores de longa distância bem treinados ( $32 \pm 6$ anos).

Estes achados sugerem que as medidas de VFC apresentam-se responsivas de maneira aguda, e ainda que uma sessão de treinamento de futsal pode gerar estímulos que ultrapassem este limiar. A atenção especial em atletas de alto rendimento em relação as alteraçóes nas medidas de VFC são fundamentais para que ajustes no planejamento possam ser realizados com o intuito de gerar um equilíbrio entre as cargas impostas e o período de recuperação evitando assim a ocorrência de lesóes e o estado de overtraining e overraching não-funcional ${ }^{32}$.

O monitoramento da VFC no futsal foi relatado pelo estudo de Oliverra et al. ${ }^{6}$, no qual demonstrou que as alteraçóes nos índices de VFC (MRR, RMSSD e HF) ocorrem com maior intensidade em atletas de futsal na pré-temporada, aumentando 
significativamente seus valores, e que durante a temporada competitiva a tendência e que ocorra a manutenção ou aumento de menor magnitude. Os autores realizaram avaliações pontuais durante esse período, indicando a ocorrência de adaptaçóes crônicas. Assim, estes resultados revelam que a resposta aguda da VFC a sessão de treinamento de futsal (queda no valor dos índices de VFC) apresenta uma dinâmica oposta as respostas crônicas (aumento nos valores dos índices de VFC). Esta diferença, possivelmente, está associada a forma como o atleta se adapta ao treinamento.

Uma explicação para o mecanismo de adaptação do atleta consiste na premissa de que o estímulo de treinamento representa um estresse psicofísico que perturba seu estado de homeostase, gerando uma queda de desempenho e fadiga temporária decorrentes de algumas respostas fisiológicas ${ }^{33}$ representadas no presente estudo pela queda dos índices de VFC. Entretanto, quando as cargas de treinamento e o processo de recuperação são organizados de maneira apropriada, o atleta atinge um estado de supercompensação, caracterizado pela melhora do desempenho, que por sua vez, também estaria relacionada a alteraçóes fisiológicas, como o aumento dos índices de VFC como expostos por Oliveira et al. ${ }^{6}$

No presente estudo, as mudanças autonômicas decorrentes da sessão de treinamento de futsal apresentaram correlaçóes positivas (moderadas) com os métodos de quantificação da CT, em especial com o método TRIMP de BANISTER et al. ${ }^{26}$, sugerindo que as medidas de VFC podem ser utilizadas como medidas de quantificação de CT em jogadores de futsal. Resultados similares foram encontrados em corredores de longa distância ${ }^{13}$, no qual os valores de VFC apresentaram concordância com os métodos de CT em especial com o método de TRIMP de Banister. Uma possível explicação pela baixa correlação com o método de Foster pode ser pelo fato deste método utilizar uma ferramenta subjetiva (PSE), sendo possível que o atleta tenha uma mudança na escolha de acordo com a expectativa do técnico ${ }^{34}$.

Vale a pena destacar que o presente estudo é o primeiro a avaliar a magnitude das mudanças cardíacas autonômicas decorrentes de uma sessão de treinamento de futsal. Consequentemente este estudo representa um primeiro passo para trabalhos futuros que visem a avaliação diária da modulação autonômica na modalidade, uma vez que a modulação autonômica varia de acordo com a intensidade do treinamento ${ }^{13}$. Contudo, vale destacar algumas limitaçôes do presente estudo como a náo padronizaçáo do controle da respiração durante a realização das medidas de VFC, podendo afetar a resposta especialmente dos índices LF e $\mathrm{HF}^{7}$ entretanto alta frequência respiratória parece não afetar LF índice, uma vez que os picos são suficientemente espaçados, não causando maiores efeitos.

Os resultados do presente estudo permitem concluir que uma sessão de treinamento realizado durante uma temporada competitiva foi capaz de gerar mudanças autonômicas significativas em jovens jogadores de futsal. Além disso, sugerese uma correlação positiva entre as medidas de VFC e CT. Esses resultados podem ser úteis para treinadores e preparadores físicos, auxiliando no ajuste das cargas de treinamento.

\section{Abstract}

\section{Acute effect of one futsal training session in cardiac autonomic modulation of young players}

The objectives of this study was to evaluate the effect of a session of futsal training on cardiac autonomic modulation (pre vs post) in young athletes. Nine U-20 male futsal players from a professional club from Sao Paulo- Brazil $(18.9 \pm 0.9$ years, weight $73.1 \pm 6.0 \mathrm{~kg}$ and $179.0 \pm 0.1 \mathrm{~cm})$ participated in this study. The training session was technical/tactical. The cardiac autonomic modulation was analyzed by heart rate variability (HRV) indices evaluate with continuous heart rate (Polar Team2 Pro) 15 minutes before and after a technical/tactical training session. Data were exported posteriorly to Kubios (2.0) program for the calculation of the indices: MRR, RMSSD, LF and HF. The paired $t$-Student test was applied to verify the pre vs post changes, besides the percentual variation $(P<0.05)$. All the indices presented significant decrease after training session and the percentual variation were: 10.7, 26.9, 4.2 and $11.3 \%$ respectively for MRR, RMSSD, LFlog and HFlog indices. The percentual of variation showed significant correlation to 
the session TRIMP session $(r=0,694)$. May be concluded that one training session was able to generate significant changes in cardiac autonomic modulation in young futsal players with variation ranging to $4.2 \%$ to $26.9 \%$. These variations are correlated positively to the training load. Therefore, these findings may help the coaches in planning the training session, monitoring the training loads.

KEYwORDS: Sport; Heart Rate Variability; Training Load; Monitoring.

\section{Referências}

1. Aubert AE, Seps B, Beckers F. Heart rate variability in athletes. Sports Med. 2003;33(12):889-919.

2. Plews DJ, Laursen PB, Stanley J, Kilding AE, Buchheit M. Training adaptation and heart rate variability in elite endurance athletes: opening the door to effective monitoring. Sports Med. 2013;43(9):773-81.

3. Buchheit M, Mendez-Villanueca A, Quod MJ, Poulos N, Bourdon P. Determinants of the variability of heart rate measures during a competitive period in young soccer players. Eur J Appl Phusiol. 2010;109(5):869-78.

4. Seiler S, Haugen O, Kuffel E. Autonomic recovery after exercise in trained athletes: intensity and duration effects. Med Sci Sports Exerc. 2007;39(8):1366-73.

5. Baumert M, Brechtel L, Lock J, Hermsdorf M, Roland W, Baier V, et al. Heart rate variability, blood pressure variability, and baroreflex sensitivity in overtrained athletes. Clin J Sport Med. 2006;16(5):412-17.

6. Oliveira RS, Leicht AS, Bishop JC, Barbero-Alvarez JC, Nakamura FY. Seasonal changes in physical performance and heart rate variability in high level futsal players. Int J Sports Med. 2013;34(5):424-30.

7. Heart hate variability: standards of measurement, physiological interpretation and clinical use. Eur Heart J. 1996;93(5):1043-65.

8. Plews DK, Laursen PB, Kilding AE, Buchheit M. Heart rate variability in elite triathletes is variation in variability the key to effective training? A case comparison. Eur J Appl Physiol. 2012;112(11):3729-41.

9. Abad CCC, do Nascimento AM, Gil S, Loturco I, Nakamura FY, Mostarda CT, et al. Cardiac autonomic control in high level Brazilian power and endurance track-and-field athletes. Int J Sports Med. 2014;35(9):772-8.

10. Boullosa DA, Tonello L, Ramos I, Silva AO, Simoes HG, Nakamura FY. Relationship between aerobic capacity and Yo-Yo 1R1 performance in Brazilian professional futsal players. Asian J Sports Med. 2013;4(3):230-4.

11. Lamberts RP, Lambert MI. Day-to-day variation in heart rate at different levels of submaximal exertion: implications for monitoring training. J Strenght Cond Res. 2009;23(3):1005-10.

12. da Silva DF, Verri SM, Nakamura FY, Machado FA. Longitudinal changes in cardiac autonomic function and aerobic fitness indices in endurance runners: a case study with a high-level team. Eur J Sport Sci. 2014;14(5):443-51.

13. Saboul D, Balducci P, Millet G, Pialoux V, Hautier C. A pilot study on quantification oftraining load: the use of HTV in training practice. Eur J Sport Sci. 2016;16(2):172-81.

14. Alvarez JCB, Soto VM, Alvarez VB, Vera JG. Match analysis and heart rate of futsal players during competition. J Sports Sci. 2008;26(1):63-73.

15. Castagna C, Ottavio S, Vera JG, Alvarez JCB. Match demands of professional futsal: a case study. J Sci Med Sport. 2009;12(4):490-4.

16. Drogramaci SN, Watsford ML, Murphy AJ. Time-motion analysis of international and national level futsal. J Strength Cond Res. 2011;25(3):646-51.

17. Rodrigues VM, Ramos GP, Mendes TT, Cabio CE, Melo ES, Condessa LA, et al. Intensity of official futsal matches. J Strength Cond Res. 2011;25(9):2482-7.

18. Matzenbacher F, Pasquarelli BN, Rabelo FN, Stanganelli LCR. Physiological demands of futsal competition: physical and physiological characteristics of professional players. Rev Andal Med Deporte. 2014;7(3):122-31.

19. Milanez VF, Ramos SP, Okuno NM, Boullosa A, Nakamura FY. Evidence of a nonlinear dose-response relationship between training load and stress markers in elite female futsal players. J Sports Sci Med. 2014;13(1):22-29.

20. Moreira A, de Moura NR, Coutts A, Costa EC, Kempton T, Aoki MS. Monitoring internal training load and mucosal immune responses in futsal athletes. J Strength Cond Res. 2013;27(5):1253-9.

21. Gomes RV, Moreira A, Lodo L, Nosaka K, Coutts AJ, Aoki MS. Monitoring training loads, stress, immune-endocrine responses and performance in tennis players. Biol Sport. 2013;30(3):173-80. 
22. Karvonen J, Vourimaa T. Heart rate and exercise intensity during sports activities: practical application. Sport Med. 1988;5(5):303-11.

23. Chandola T, Heraclides A, Kumari M. Psychophysiological biomarkers of workplace stressors. Neurosci Biobehav Rev. 2010;35(1):51-7.

24. Borg GAV, Hassmen P, Langerstrom M. Perceived exertion in relation to heart rateand blood lactate during arm and leg exercise. Eur J Appl Physiol. 1987;56(6):679-85.

25. Foster C, Florhaug JA, Franklin J, Gottschall L, Hrovatin LA, Parker S, et al. A new approach to monitoring exercise training. J Strenght Cond Res. 2001,15(1):109-15.

26. Banister EW. Modeling elite athletic performance. In: Green HJ, McDougal JD, Wenger HA, editores. Physiological testing of elite athletes. Champaign: Human Kinetics; 1991. p. 403-24.

27. Karvonen MJ, Kentala E, Mustala O. The effects of training on heart rate: a longitudinal study. Ann Med Exp Biol Fenn. 1957;35(3):307-15.

28. Sztajzel J. Heart rate variability: a noninvasive electrocardiographic method to measure the autonomic nervous system. Swiss Med Wkly. 2004;134(35-36):514-22.

29. Hopkins WG. A scale of magnitudes for effect statistics [Internet]. [local desconhecido]: A new view of statistics; 2002 [citado em 2018 mar 2018]. Disponível em: <https://goo.gl/HuDUcX>.

30. Vanderlei LCM, Patre CM, Hoshi RA, Carvalho TD, Godoy MF. Noçóes básicas de variabilidade da frequência cardíaca e sua aplicabilidade clínica. Rev Bras Cir Cardiovasc. 2009;24(2):205-17.

31. Raczak G, Pinna GD, la Rovere MT, Maestri R, Szymanowicz LD, Ratkowski W, et al. Cardiovagal response to acute mild exercise in young healthy subjects. Circ J. 2005;69(8):976-80.

32. Purvis D, Gonsalves S, Deuster PA. Physiological and psychological fatigue in extreme conditions: overtraining and elite athletes. PM R. 2010;2(5):442-50.

33. Fry RW, Morton AR, Keast D. Periodisation of training stress: a review. Can J Sport Sci. 1992;17(3):234-40.

34. Borresen J, Lambert MI. The quantification of training load, the training response and the effect on performance. Sports Med. 2009;39(9):779-95.

\begin{tabular}{r|r} 
ENDEREÇo & \\
Ana Carolina Paludo & \\
Escola de Educação Física e Esporte da & Recebido para publicação: 11/o6/2015 \\
Universidade de São Paulo & Revisado: 14/10/2015 \\
O5508-030 - São Paulo - SP - BRASIL & Aceito: 29/12/2015 \\
e-mail: anacarolinapaludo@usp.br & \\
& \\
\hline
\end{tabular}

